\title{
Influence of geometry of nanocrystals on the Raman spectra
}

\author{
A. V. Igo \\ Ulyanovsk State University, 432017, L. Tolstoy 42, Ulyanovsk, Russia \\ igoalexander@mail.ru
}

PACS 78.30-j; 61.46-w

DOI 10.17586/2220-8054-2018-9-1-132-135

\begin{abstract}
It is shown that Raman spectra of nanocrystals with complex geometric shape can acquire additional broadening. In order to describe complex geometric shapes of nanocrystals in terms of its influence on the Raman spectrum, a parameter was introduced for the shape - roughness parameter. The roughness is defined as a relative parameter of the presence of additional volume on the faces of the cubic nanocrystal. The calculations for additional broadening of the Raman spectral line were made for 3-10 nm nanodiamonds.
\end{abstract}

Keywords: nanodiamonds, Raman spectra, nanocrystal.

Received: 26 April 2017

Revised: 11 October 2017

\section{Introduction}

Nanocrystals and technical systems based on them are increasingly being used in practice. Special physical and optical properties of nanoparticles are due to quantum size effects.

The size of a nanocrystal influences the Raman spectrum by spatial limitation of optical phonons (phonon confinement). This influence is studied in detail and described in literature [1]. In particular, it was found that the Raman spectrum of nanodiamond is shifted to the lower frequencies and the spectral line acquire additional broadening [2]. Together with the X-ray diffraction method, Raman spectra analysis has become almost a routine method for determining the size of the nanocrystals. This has become possible by obtaining empirical relations between the parameters of the spectral line and the size of the nanoparticles [1]. According to the relations, the frequency $\omega$ of the spectral peak and the width of the peak $\Gamma$ depend on the size of a nanocrystal as:

$$
\omega=\omega_{0}+\frac{A}{d^{1.5}} ; \quad \Gamma=\Gamma_{0}+\frac{B}{d^{1.5}} .
$$

Here, $\omega_{0}$ and $\Gamma_{0}$ are the frequency and the width of the peak in the large crystal, and $d$ is the size of the nanocrystal. The exponent at $d$ depends on the type of a nanocrystal, for example, in diamond nanocrystals it equals one [3].

The theory of phonon confinement describes the observed phenomena in the spectra of nanoparticles, nevertheless, theoretical studies aimed at improving the model are in progress. The studies are concerning the relation between the size of nanocrystals and the peak shift [4-6] and the width of the peak $[4,5,7]$.

It was shown by the method of numerical simulation [7], that the geometric form of nanocrystalline particles significantly influences the width of the Raman spectral line. This means that the empirical coefficient B in the equation (1) depends on a shape parameter of the nanocrystal. A mathematical model of the nanoparticles with the complex shape and the parameter of the geometric shape for the particle were introduced in [7].

This study summarizes the approach used in [7] and presents calculations of the spectral line broadening for some values of the shape parameter. An analytical equation correlating the spectral line width with the size and the shape parameter (roughness) of a nanocrystal is proposed.

\section{Model}

The intensity of Raman radiation in the model [7] is a sum of intensities from the core volume $V=d^{3}$ of a nanocrystal and from the additional volume $\Delta V$ (Fig. 1).

In accordance with the quantum mechanical approach, an optical phonon in the center of the Brillouin zone, when confined within the length $d$, acquires a wave vector with the minimum value of $q_{d}=2 \pi / d$. According to the dispersion law, this changes the frequency of the phonon to $\Omega\left(q_{d}\right)$, the value different from the phonon frequency $\Omega(0)$ in a large crystal. 


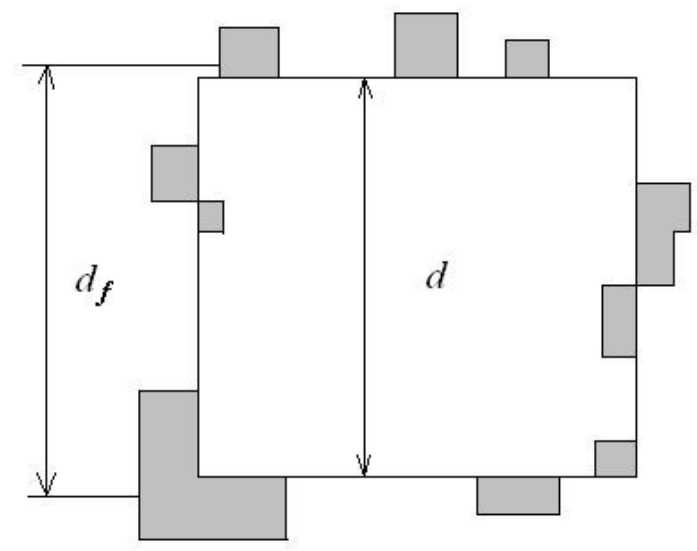

FIG. 1. Nanocrystal of size $d_{f}$. The nanocrystal has main volume $V=d^{3}$ and additional volume $\Delta V$, corresponding to shaded area

The additional volume $\Delta V$ can be represented as a sum of volumes of cubes with edge length of $d / n$ ( $n$ is an integer number). Given $s_{n}$ is the probability to find the additional volume $(d / n)^{3}$ at any face of the main cube, the total additional volume is:

$$
\Delta V=\sum_{n=2}^{N} 6 s_{n} n^{2}(d / n)^{3} \cong 6 s d^{3} \sum_{n=2}^{N}(1 / n) .
$$

The equation (2) assumes a uniform distribution of the probability to find the additional volume for different sizes on the face of the cube, and this probability is denoted by s. Each of the volumes will contribute to scattering of light on the combination frequency $\omega-\Omega\left(q_{n}\right)$, where $q_{n}=2 \pi n / d$. Here, $\omega$ is the frequency of the incident light. The parameter $s$ in (2) may be referred as the roughness parameter of the nanoparticle, since it characterizes the additional volume which resides on the faces of the cube nanoparticle.

Thus, the Raman spectrum of a particle of size $d$ has two contributions: the first one from the main particle volume, $d^{3}$, and the second one from the additional volume generated by geometrical irregularities of the particle with the roughness $s$ :

$$
I(\omega)=\frac{(\Gamma / 2)^{2}}{\left[\omega-\Omega\left(q_{d}\right)\right]^{2}+(\Gamma / 2)^{2}}+\sum_{n=2}^{N} \frac{6 s(\Gamma / 2)^{2}(1 / n)}{\left[\omega-\Omega\left(q_{n}\right)\right]^{2}+(\Gamma / 2)^{2}} .
$$

The spectrum (3) has a distinctive asymmetrical form, additionally broadened and shifted towards lower frequencies. The value of the asymmetry, and other parameters of the modified spectral line are determined by the dispersion function of the optical phonons. Here we used the dispersion function $\Omega\left(q_{n}\right)$ from the model of the cubic crystal lattice with additional constraints [8].

\section{The spectral line width calculation}

The total volume of the particle shown in Fig. $1, V_{f}=d^{3}+\Delta V$, roughly corresponds to the particle size $d_{f}=V_{f}^{1 / 3}$, and the corresponding Lorentzian intensity of the scattered light is:

$$
I(\omega)=\frac{\left(\Gamma_{f} / 2\right)^{2}}{\left[\omega-\Omega\left(q_{f}\right)\right]^{2}+\left(\Gamma_{f} / 2\right)^{2}},
$$

where $q_{f}=2 \pi / d_{f}$.

An actual spectrum is significantly asymmetric and this simple expression does not describe it in full. For the low-frequency wing of the Lorentzian alone $\left(\omega<\Omega\left(q_{f}\right)\right)$ one can equate (3) and (4) for the same volume:

$$
\frac{\left(\Gamma_{f} / 2\right)^{2}}{\left[\omega-\Omega\left(q_{f}\right)\right]^{2}+\left(\Gamma_{f} / 2\right)^{2}} V_{f}=\frac{(\Gamma / 2)^{2}}{\left[\omega-\Omega\left(q_{d}\right)\right]^{2}+(\Gamma / 2)^{2}} d^{3}+\sum_{n=2}^{N} \frac{6 s d^{3}(\Gamma / 2)^{2}(1 / n)}{\left[\omega-\Omega\left(q_{n}\right)\right]^{2}+(\Gamma / 2)^{2}} .
$$

The equation must be true for any frequency $\omega<\Omega\left(q_{f}\right)$. Let consider the point $\omega=\Omega\left(q_{d}\right)-\Gamma / 2$. In this case, the equation can be solved for the width of the spectral line $\Gamma_{f}$ of the particle of a volume $V_{f}$ with a 
roughness parameter $s$. Given the notations:

$$
\beta=\sum_{n=2}^{N}(1 / n) ; \quad \gamma=\sum_{n=2}^{N} \frac{(1 / n)}{\left(2 \frac{\Omega\left(q_{d}\right)-\Omega\left(q_{n}\right)}{\Gamma}-1\right)^{2}+1} ; \quad V_{f}=d^{3}(1+6 s \beta),
$$

we obtain:

$$
\left(\Gamma_{f} / 2\right)^{2}=\left(\Omega\left(q_{d}\right)-\Omega\left(q_{f}\right)-\Gamma / 2\right)^{2} \frac{1 / 2+6 s \gamma}{1 / 2+6 s(\beta-\gamma)} .
$$

The sizes $d$ and $d_{f}$ are nearly equal (Fig. 1). By expanding $\Omega\left(q_{d}\right)$ and $\Omega\left(q_{f}\right)$ in Taylor series in the vicinity of $q_{f}=2 \pi / d_{f}$ and leaving only the first non-zero term of the expansion, in view of $d_{f}=V_{f}^{1 / 3}=d(1+6 s \beta)^{1 / 3}$, we get:

$$
\Omega\left(q_{d}\right)-\Omega\left(q_{f}\right)=\left(\frac{\alpha}{d}\right)^{2}\left(1-\frac{d^{2}}{d_{f}^{2}}\right)=\left(\frac{\alpha}{d}\right)^{2}\left(1-\frac{1}{(1+6 s \beta)^{2 / 3}}\right) .
$$

The factor $\alpha$ describing the dispersion of optical phonons in the vicinity of $q_{d}$ depends on a type of nanocrystal. Finally, the width of the line is:

$$
\Gamma_{f}=\left[\Gamma+2\left(\frac{\alpha}{d}\right)^{2}\left(1-\frac{1}{(1+6 s \beta)^{2 / 3}}\right)\right]\left(\frac{1 / 2+6 s \gamma}{1 / 2+6 s(\beta-\gamma)}\right)^{1 / 2} .
$$

Calculations of $\Gamma_{f}$ were made for nanoparticles of diamond with the size $d$ in the range of 10-20 lattice constants and the roughness parameter $s$ in the range from 0.01 to 0.2 (Fig. 2). The additional width of the spectral line varies from 0 to $7 \mathrm{~cm}^{-1}$.

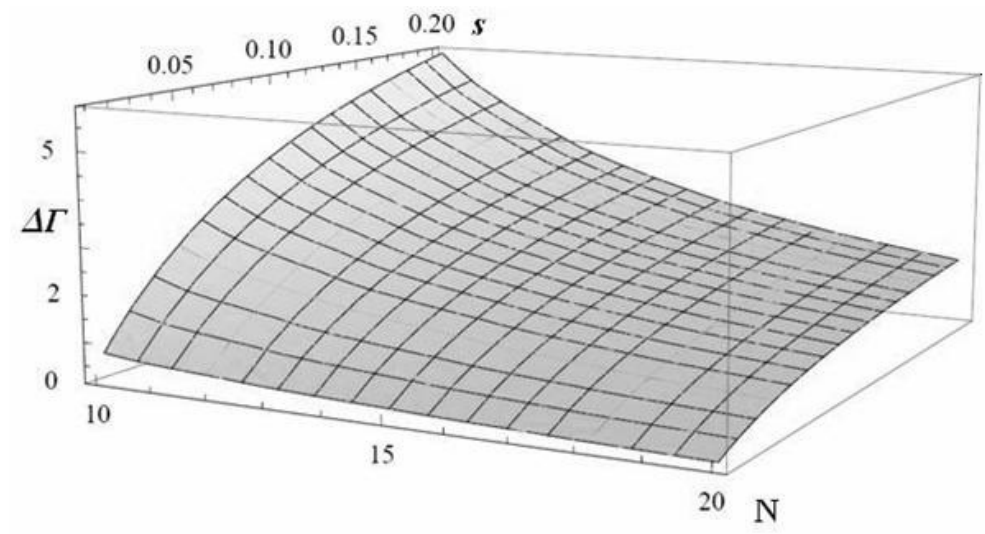

FIG. 2. Additional width of the Raman spectral line of a diamond nanoparticle with size from 10 to 20 lattice constants and a roughness $s$ from 0.01 to 0.2

In many cases of practical importance, it is convenient to deal with spherical crystalline nanoparticles. One can assume that there is an additional volume $\Delta V \approx 0.4 d^{3}$ on the faces of the cube inscribed in such a particle. The estimation of the roughness parameter with (5) and (2) gives $s=\Delta V /\left(6 d^{3} \beta\right) \approx 0.07$.

The additional broadening of the spectral line can be taken into account when comparing the theory with an experiment. In [7] such a comparison was performed for spherical nanocrystals of silicon. The additional broadening was added to the theoretical curve obtained in [4]. In this case, the total width of the Raman spectral line more accurately describes the experimental data.

The equation (7) can be simplified for a case when $s$ is small. Assuming $6 s \beta \ll 1$ and $\beta>\gamma$, we obtain:

$$
\Gamma_{f} \approx \Gamma+8 \alpha^{2} s \frac{\beta}{d^{2}} .
$$

Thus, the additional width is proportional to the value of dispersion of the optical phonons and the roughness of a nanocrystal. The size dependence is determined by the value of $\beta / d^{2}$. As seen from (5), $\beta$ is a function of particle size expressed in units of lattice period, $N=d / a$. Direct calculation shows that for a diamond nanocrystal $2-10 \mathrm{~nm}, \beta$ can be approximated by a power law $\beta(d) \sim d^{k}$, where $k$ is in the range from 0.5 to 0.3 . The obtained equation (8) is in a good agreement with the empirical equation (1). 


\section{Conclusion}

The analytical equation for a width of a Raman spectral line in nanocrystals of a complex shape is obtained. The additional broadening of the line is calculated for the nanodiamonds of 3-10 nm in size. This broadening depends on the shape parameter and on the size of the nanocrystal. The proposed analytical equation for the low roughness approximation is in good agreement with the known empirical relation.

\section{References}

[1] Arora A.K., Rajalakshmi M., Ravindran T.R., Sivasubramanian V. Raman spectroscopy of optical phonon confinement in nanostructured materials. J. Raman Spectrosc., 2007, 38, P. 604-617.

[2] Mikov S.N., Igo A.V., Gorelik V.S. Raman scattering of light by diamond quantum dots in a potassium bromide matrix. Phys. Solid State, 1995, 37, P. 1671-1673.

[3] Yoshikawa M., Mori Y., Obata H., Maegawa M., Katagiri G., Ishida H., Ishitani A. Raman scattering from nanometer-sized diamond. Appl. Phys. Lett., 1995, 67, P. 694-696.

[4] Faraci G., Gibelisco S., Russo P., Pennisi A.R., Faraci C. Quantum size effects in Raman spectra of Si nanocrystals. J. Appl. Phys., 2011, 109, P. 074311.

[5] Volodin V.A., Sachkov V.A. Improved model of optical phonon confinement in silicon nanocrystals. J. Exp. Theor. Phys., 2013, 116, P. 87-94.

[6] Meilakhs A.P., Koniakhin S.V. New explanation of Raman peak redshift in nanoparticles. Superlatt. Microstruct., 2017 , 110, P. $319-323$.

[7] Igo A.V. Inhomogeneous size broadening of the Raman scattering spectral line in nanocrystals. Optics and Spectroscopy, 2016, 120(4), P. 529-533.

[8] Gorelik V.S. Idealized models of crystal lattices and the spectra of real crystals. Tr. Fiz. Inst. AN, 1987, 180, P. 87-126. 